\title{
Analysis of non-pharmaceutical interventions impacts on COVID-19 pandemic in Iran
}

\author{
Sara Saadatmand $\cdot$ Khodakaram Salimifard (i) $\cdot$ Reza Mohammadi
}

Received: 4 February 2021 / Accepted: 30 November 2021 / Published online: 11 January 2022

(C) The Author(s), under exclusive licence to Springer Nature B.V. 2021

\begin{abstract}
The COVID-19 pandemic shows to have a huge impact on people's health and countries' infrastructures around the globe. Iran was one of the first countries that experienced the vast prevalence of the coronavirus outbreak. The Iranian authorities applied various non-pharmaceutical interventions to eradicate the epidemic in different periods. This study aims to investigate the effectiveness of non-pharmaceutical interventions in managing the current Coronavirus pandemic and to predict the next wave of infection in Iran. To achieve the research objective, the number of cases and deaths before and after the interventions was studied and the effective reproduction number of the infection was analyzed under various scenarios. The SEIR generic model was applied to capture the dynamic of the pandemic in Iran. To capture the effects of different interventions, the corresponding reproduction number was considered. Depending on
\end{abstract}

S. Saadatmand · K. Salimifard $(\bowtie)$

Computational Intelligence \& Intelligent Optimization

Research Group, Persian Gulf University, Bushehr 75169, Iran

e-mail: salimifard@pgu.ac.ir

S. Saadatmand

e-mail: sara.saadatmand@mehr.pgu.ac.ir

R. Mohammadi

Department of Operation Management, Amsterdam

Business School, University of Amsterdam, Amsterdam,

Netherlands

e-mail: a.mohammadi@uva.nl how people are responsive to interventions, the effectiveness of each intervention has been investigated. Results show that the maximum number of the total of infected individuals will occur around the end of May and the start of June 2021. It is concluded that the outbreak could be smoothed if full lockdown and strict quarantine continue. The proposed modeling could be used as an assessment tool to evaluate the effects of different interventions in new outbreaks.

Keywords COVID-19 - Pandemic modeling - SEIR model $\cdot$ Non-pharmaceutical interventions $\cdot$ Nonlinear dynamics

\section{Introduction}

Epidemic diseases have always been a threat to human life. Some of them are prevalent throughout various continents and countries and become pandemic. Black Death and 1918 influenza pandemic are among the deadliest pandemics in human life and caused millions of deaths in past centuries [1]. Except for its influence on humans' fatality, the epidemic can have irreparable losses in nations' economics and industries. Among recent epidemic illnesses, such as Ebola, yellow fever and Zika, the novel coronavirus has attracted a lot of attention due to its infections and death numbers. The novel Coronavirus (COVID-19) is a new type of 
coronaviruses family that can cause different diseases, which are frequently respiratory infections. The severity of illnesses can vary from common cold to the Middle East respiratory syndrome (MERS) and severe acute respiratory syndrome (SARS) [2]. This virus first appeared in Wuhan, Hubei Province of China, on December 29th, 2019, and then spread rapidly across the world. On March 11th, 2020, the World Health Organization (WHO) characterized this illness as a pandemic [3].

The COVID-19 epidemic has had a huge impact on people's health, economics and countries' infrastructures such as communication, transportation, energy, and public health and due to that the government have applied rigid health interventions to reduce its impacts. Health interventions refer to those activities and actions, which protect human health and mitigate the disease prevalence, by decreasing disease duration and severity, and function loss [4]. One of the classifications for health interventions is to divide them into two classes of pharmaceutical and nonpharmaceutical interventions. The first class contains vaccines and antiviral drugs [5] and the second one includes government interventions like postponing and canceling the events, social distancing, travel restrictions, and home quarantine [6].

Lockdown is among the non-pharmaceutical interventions used vastly in most of the countries in different periods of times to control the virus spread. The main goal of this strategy is to decrease the number of infected individuals so the hospital overcapacity that causes more deaths would be prevented. Merriam-Webster dictionary defines lockdown as a situation that is not permanent and applied by governmental authorities in times of epidemic to make people stay home and limit outside activities [7]. Full and partial lockdown are two common lockdown strategies imposed by government on communities around the world to reduce the effect of the pandemic. During the COVID-19 outbreak, several countries such as China, Italy, Spain, and France applied strict complete lockdown while Singapore, Russia, and Morocco used partial lockdown in which some of the social and economic activities were allowed [8].

To analyze and predict the behavior of viruses in a community is a vital issue to provide a comprehensive view for policymakers and assist them in making decisions for interventions such as lockdown and vaccine. Mathematical compartmental modeling is a quantitative approach that is based on the system of differential equations that capture the dynamic of epidemic transmission [9]. SIR (susceptible-infectious-recovered) model is one of the most used epidemic compartmental models [10], especially to study the COVID-19 prevalence [11]. By presenting two parameters, this model proposes a more clear and understandable tool in comparison to its more complex derivatives [12]. Mathematical SIR models have been used in many articles so far to make predictions about the novel Coronavirus and evaluate authorities' interventions. Malavika et.al [13] applied this model to estimate the number of infected cases and predict the time of pick in India. They couldn't find any evidence that proves the relationship between reducing the number of infected persons and applying lockdown. [14] Used SIR and machine learning modeling to forecast the pandemic behavior in the next 700 days under three scenarios: no actions, lockdown, and new medicines in KSA. The results indicated that containment strategies such as lockdown and quarantine alone are inadequate to eradicate the pandemic. Sadurni and Luna-Acosta [15] represented an improved version of the SIR model. They considered parameters' variation with time and migration of susceptible and infected individuals. Authors applied a generalized logistic model to fit Mexico's COVID-19 data and achieved the bifurcation diagram for a discretized version of the generalized logistic model.

To predict the number of cases and the second wave of epidemic in Iran Ghanbari [16] developed a SIR model with two compartments for infected people: $I_{1}(t)$ (infected individuals with strong immunity system), and $I_{2}(t)$ (infected individuals with weak immunity system). The results revealed that the second wave in Iran would be more intense than the previous one. Tutsoy et.al [17] extended a new model from SIR called SpID to forecast fatality in Turkey. [18] Used a modified SIR model with global pandemic data to predict the virus spread globally while considering environmental factors like temperature and humidity. To predict the epidemic trend in Italy Wangping et.al [19] applied an extended SIR model to the time series data. [20] Estimated the basic reproduction number $\left(\mathcal{R}_{0}\right)$ by using the compartmental SIR model in India. To evaluate the amount of infected population and deaths in Sweden Qi et.al [21] applied SI, SIR, and SID epidemic models. 
Among SIR extensions the classical SEIR is one of the most traditional epidemic model which indicates the progression of virus in population by four states: Susceptible(s), Exposed (E), Infectious (I), and Recovered (R) [22]. This model has been used in several articles to predict and evaluate the spread of novel coronavirus pandemic. Kwuimy et.al. [23] used a nonlinear SEIR model to analyze the dynamic behavior of the COVID-19 pandemic in South Korea. The genetic algorithm was applied to approximate the model parameters. [22] Studied the management strategies such as lockdown with adaption of SEIR model in the US. To forecast the COVID-19 evaluation in Hubei Province [24] applied an extended SEIR model with quarantine and hospitalized compartments under different scenarios and the particle swarm optimization (PSO) algorithm to estimate the model parameters. [25] proposed a new SEIR model with two more components: quarantined and insusceptible. Their improved model captured the prevalence of the virus in the US and forecasted the future trend. Jayrold et al. [26] used the extension of SEIR to estimate the effective reproduction number for several countries. [27] Developed a SEIR model with the effect of time delay for India. Arghya et al. [28] applied SEIR epidemiological model to predict the peak of infected cases and explore the impact of social distancing and testing-quarantining on virus propagation.

Iran was one of the first countries which experienced the vast spread of this virus. The first confirmed cases in Iran were reported on February 19th, 2020, in city of Qom [29]. Since then, the Iranian government have used several policies and strategies based on a number of confirmed cases and deaths in provinces. According to the confirmed cases statistics, each city is categorized in one of the blue, yellow, orange, and red categories which sequence demonstrates the severity of the pandemic from low to high. Based on the literature, no article has evaluated the effectiveness of government interventions on COVID-19 prevalence in Iran.

In the following first the epidemic SEIR model will be described, then the impact of lockdown on number of infected individuals and deaths will be evaluated. Then, based on the official reported data the parameters will be estimated and the model will forecast the next peak of out-break in Iran. Finally, the conclusion will be presented.

\section{Epidemic modeling}

The advent and outbreak of epidemic have always been a noticeable issue in mathematical modeling in order to predict the virus spread and evaluate the impact of policies and interventions. Mathematical epidemic models are vigorous tools that let healthcare policy makers anticipate various changes that occur by virus mutation, vaccination, quarantine and lockdown in the number of infected individuals and mortality, moreover, make efficient decisions. The first epidemic model was proposed by Daniel Bernoulli (1700-1782) to oppose smallpox, which was one of the fatal diseases of that era. Soon after that different people started to develop epidemic models and finally Kermack and McKendrick presented their compartmental model, which divided the population into three categories: S, I, R [30].

Compartmental models simulate the spread of pathogens and investigate the transmission dynamics of contagious disease in a host population [31]. They place population into distinctive classes and people move among them at diverse rates. The model that had been presented by [32] is considered as the basic model in epidemic which later various compartments such as exposed, deceased, and asymptotic were added to it.

\subsection{The SEIR model}

In the basic model which was introduced by Kermack and McKendrick [32] the host population is divided into three classes: susceptible, infected, and recovered that are called S, I, and R in contractions. As shown in Fig. 1, the SEIR model adds another component to SIR, which is E and refers to the exposed population. Susceptible are those who have not had the disease before but are likely to get it and are transmitted to E as soon as they are infected. Exposed individuals are those who have contracted the virus and are not being confirmed to be infected but can transmit the disease to other people. Infected are people who have the disease

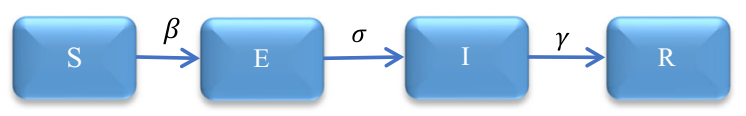

Fig. 1 The generic SEIR model dividing the population into four compartments: susceptible, exposed, infected, and recovered 
and the same as the exposed can transmit it to susceptible. The time period that people are in infected class is assumed as the infectious period. Recovered are those who got the disease and got well and have immunity to the virus or died. Equation (1) denotes the total population and its compartments at time $t$.

$N=S(t)+E(t)+I(t)+R(t)$

The SEIR model by applying a system of ordinary differential equations (ODE), presents a constant model with continuous time that has three rates: $\beta, \sigma$ and $\gamma$. The assumption of constant population and model ODEs are shown in Eqs. (2) and (3), respectively. $\beta$ is the transmission rate from susceptible to exposure and called the infection rate. The incubation rate, $\sigma$, is the rate of latent individuals becoming infectious and $\gamma$ is the transition rate from infected to recovered and called recovery rate. All of these parameters are constant. Parameters $S_{0}, E_{0}, I_{0}$, and $R_{0}$ in Eq. (3) demonstrate the initial conditions of the model.

$\frac{d N}{d t}=\frac{d S}{d t}+\frac{d E}{d t}+\frac{d I}{d t}+\frac{d R}{d t} \equiv 0$

$$
\left\{\begin{array}{l}
\frac{d S}{d t}=-\frac{\beta I S}{N} \\
\frac{d E}{d t}=\frac{\beta I S}{N}-\sigma E \\
\frac{d I}{d t}=\sigma E-\gamma I \\
\frac{d R}{d t}=\gamma I \\
S(0)=S_{0} ; E(0)=E_{0} ; I(0)=I_{0} ; R(0)=R_{0}
\end{array}\right.
$$

\subsection{The basic reproduction number}

The basic reproduction number, which is indicated by $\mathcal{R}_{0}$ is the estimated number of secondary infected individuals produced by a single infected person in a completely susceptible population [33]. $\mathcal{R}_{0}$ is one of the most basic and common ratios to study and explore the spread of an infectious disease among a host population. As it is denoted in Eq. (4), $\mathcal{R}_{0}$ is the ratio of infection rate to the recovery rate. If this ratio equals three, it means that one infected person is likely to transmit the disease to almost other three individuals. In mathematical infection models like SEIR, three different possibilities exist for values of $\mathcal{R}_{0}$ : (1) when $\mathcal{R}_{0}<1$, each infected person causes less than one new infection, the infection will not be spread. (2) When $\mathcal{R}_{0}=1$, the disease will remain alive in the community but will not lead to an epidemic. (3) When $\mathcal{R}_{0}>1$, the disease will spread among the population and each infected person will transmit the infection to more than one new individual. Normally, the greater value of $\mathcal{R}_{0}$ causes more intense epidemic prevalence.

$\mathcal{R}_{0}=\frac{\beta}{\gamma}$

\section{Modeling the outbreak in Iran}

In this section, the model parameters are set to capture the outbreak in Iran. Then, the next wave of the infection is predicted. The details are given in the following sub-sections.

\subsection{Data source}

The data for this study were obtained from publicly available dataset of COVID-19 provided by the Center for Systems Science and Engineering (CSSE) at Johns Hopkins University [34]. This dataset consists of daily number of confirmed cases, recovered individuals, and deaths of many countries which are officially announced by health authorities' of each country. Data included in this analysis are those from February 19, 2020, to January 21, 2021.

\subsection{Model fitting and parameters estimation}

The initial conditions of the COVID-19 epidemic in Iran which was firstly reported on February 19th, 2020, consist of two infected cases and zero recovered. Due to the insufficiency of COVID-19 test kits, the reported number of cases was much lower than the actual number. It could influence the prediction model to produce wrong results. So, to have a more realistic and accurate prediction, it was decided to consider initial conditions based on the reported data on August 1st, 2020. Accordingly, the initial conditions are as follows: $\quad \mathrm{S}(0)=81,876,676, \quad \mathrm{E}(0)=1,533,760$, $\mathrm{I}(0)=306,752, \mathrm{R}(0)=282,812$. As it is suggested [35], the initial number of exposed individuals is assumed to be five times the number of reported infected on the first day which here is August $1^{\text {st }}, 2020$. 
In order to fit the model to actual data, a solver for the ODEs and an optimizer to find the optimal values for the parameters are required. To solve the ODE system, the Livermore solver of ordinary differential equations with automatic method switching (LSODA) [36] was applied. The LSODA method automatically chooses methods for stiff or non-stiff problems and assumes a full or banded Jacobian. Accordingly, using this algorithm, it is not necessary to define whether the problem is stiff or non-stiff; the solver will choose the proper method automatically. The LSODA algorithm uses the non-stiff method initially and then, it dynamically investigates the data for selecting the suitable method [37]. The LSODA algorithm was originally implemented using the FORTRAN programing language. To solve the ODEs in this article, the $\mathrm{R}$ programing language was applied. The $\mathrm{R}$ function LSODA provides an interface to the FORTRAN ODE solver of the same name.

To optimize the value of parameters and minimize the difference between obtained values from ODEs and actual data, the Limited Memory BroydenFletcher-Goldfarb-Shanno with simple bounds (LBFGS-B) optimization algorithm was used [38, 39]. The L-BFGS-B is the extension of the limited memory algorithm with the ability to enforce bounds on the variables for solving large-scale nonlinear optimization problems [40]. In the L-BFGS-B algorithm, the goal is to minimize a nonlinear function of $n$ variables [41]. L-BFGS-B is a gradient projection-based optimization algorithm that does not require knowledge about the Hessian matrix of $f$. The minimization problem is shown in Eq. (5) and Eq. (6):

$\min f(x)$

$l \leq x \leq u$

where the objective function $f: \mathbb{R}^{n} \rightarrow \mathbb{R}$ is a nonlinear function subject to some boundary constraints. This function minimizes the variation of modeling errors. In this article, $x$ refers to $\beta$ (infection rate) and $\gamma$ (recovery rate) and $f(x)$ find the optimal values for these parameters by applying the L-BFGS-B algorithm. Since there are two parameters to be optimized ( $\beta$ and $\gamma$ ), then $n=2$. The initial values for $\beta$ and $\gamma$ in the L-BFGS-B method were set to 0.5 and 0.5 , respectively. In Eq.REF_Ref70624569 \h) $l$ and $u$ demonstrate lower and upper bounds on the variables, respectively. Based on the variation range of the parameters, the lower $(l)$ and upper $(u)$ bounds for both $\beta$ and $\gamma$ are considered to be 0 and 1 , respectively. The algorithm begins with an initial value of $x$ and then continues to find better values for $x$ such that the resulting $f$ is minimized. The interested reader is referred to [42].

To measure the discrepancy between the actual data and predicted values (the variation of modeling errors), the residual sum of squares (RSS) was used Eq. (7). In this article, the $R S S(\beta, \gamma)$ calculates the sum of residual squares based on the reported data (Johns Hopkins GitHub dataset) and the predicted values of COVID-19 infected $(I)$ and recovered $(R)$ cases which calculated by applying LSODA algorithm on ODEs. To make the prediction more accurate, the objective function $f(x)$ by using the L-BFGS-B algorithm finds the optimal values for both $\beta$ and $\gamma$ such that the residual sum of squares (RSS) is minimized; therefore $f(x)=R S S(\beta, \gamma)$.

Generally, a smaller RSS indicates that the model can make better parameter estimation.

$$
\begin{aligned}
R S S(\beta, \gamma)= & \sum_{n=1}^{m}\left[\left(I_{\text {observed }}^{n}-\tilde{I}_{\text {predicted }}^{n}\right)^{2}\right. \\
& \left.+\left(R_{\text {observed }}^{n}-\tilde{R}_{\text {predicted }}^{n}\right)^{2}\right]
\end{aligned}
$$

where $m$ is the number of days between August 1, 2020, and January 21, 2021, and $I^{n}=I\left(t_{n}\right)$, and $R^{n}=R\left(t_{n}\right) \cdot I^{n}$ and $R^{n}$ denote the reported values of infected and recovered COVID-19 cases in day $n$ (Johns Hopkins GitHub dataset) while, $I^{n}$ and $\tilde{R}^{n}$ are the predicted values of infected $(I)$ and recovered $(R)$ individuals in day $n$ by applying the LSODA on the ODEs as stated above [43]. Since in the SEIR model each compartment has a specific incoming and outgoing rate [44], for each day $d$, the number of infected and recovered in $d$ is calculated using Eqs. (8) and (9).

$I(d)=I(d-1)+\widehat{I}(d)-\widehat{R}(d)$

$R(d)=R(d-1)+\widehat{R}(d)$

where $\widehat{I}(d)$ represents the number of new infections reported on day $d$ and $\widehat{R}(d)$ refers to number of new recovered individuals on day $d$. Figure 2 demonstrates the fitted model against actual data.

The transmission rate from exposure to infected compartment is $\sigma=1 / d$, where $d$ is the average time 


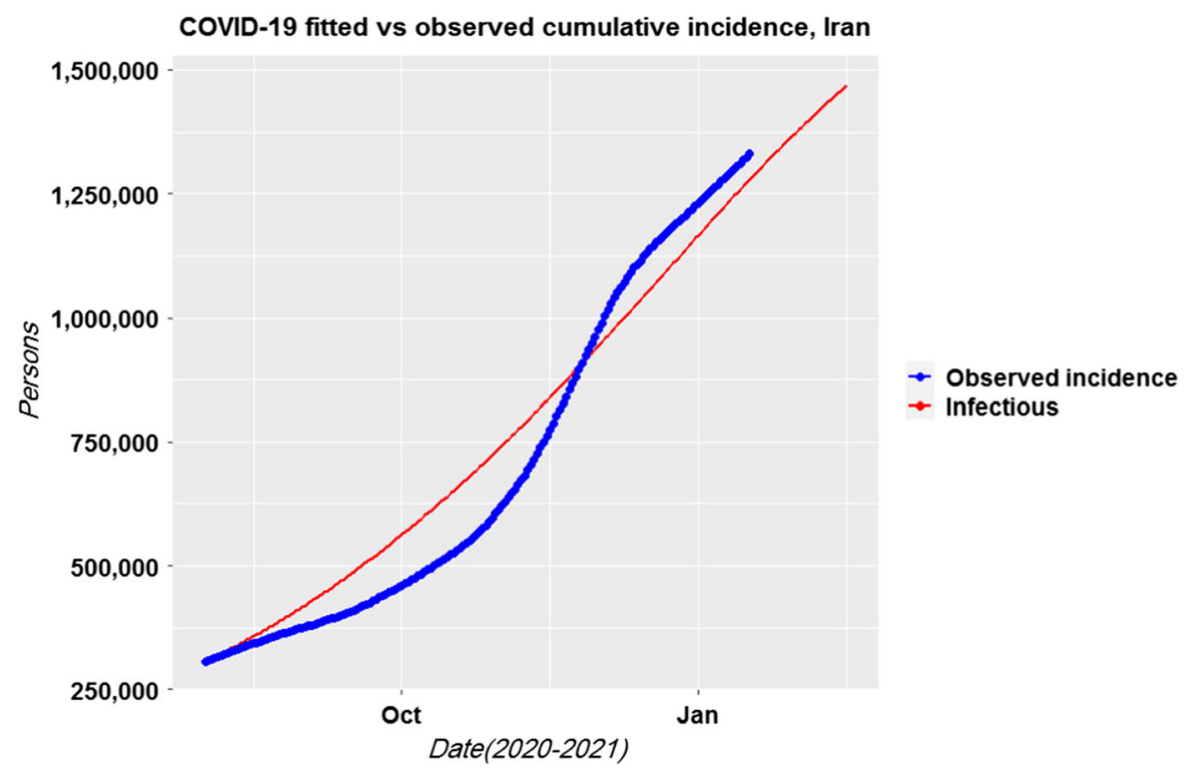

Fig. 2 Graphical representation of the fitted model to the officially reported the number of infected individuals of Iran from August 1, 2020, to January 21, 2021. The blue line

between exposure to the virus and symptom onset. As discussed in [45] $d$ is 5-6 days. In this study, it is set to 5.5 days; therefore $\sigma=0.1818$. The time interval of infected individuals for parameter estimation is considered from August 1, 2020, to January 21, 2021.

The values of $\beta$ and $\gamma$ parameters are estimated using the aforementioned L-BFGS-B method. They are, therefore, estimated to be 0.0612 and 0.0505 , respectively. Using the estimated values, the basic reproduction number $\left(\mathcal{R}_{0}\right)$ for Iran is calculated in Eq. (10).

$\mathcal{R}_{0}=\frac{\beta}{\gamma}=\frac{0.0612}{0.0505}=1.2120$

The model is implemented in $\mathrm{R}$ and runs on a Windows 10 computer with Core i7-2.6 GHz CPU with $12.0 \mathrm{~GB}$ memory. As it is demonstrated in Eq. (10) the $\mathcal{R}_{0}$ is more than unity, which means that the epidemic is spreading among the population and causing illness and death, so there is an urgency for governmental intervention to eradicate it.

\subsection{Prediction of the new wave of infection}

As illustrated in Fig. 3, Iran has passed three waves of the outbreak which happened in April, mid-July to mid-September, and December 2020. The speed and represents the daily cases, while the red line corresponds to the best fit of the SEIR model to the actual data

severity of the waves have been increasing as the days passed. As shown in Fig. 3, compared to the interval between the first and second waves, the time interval between the second and third waves has been reduced.

In this section, the SEIR model is applied to predict the peak of the total number of infected individuals in Iran. The available data from August 1, 2020, to January 21, 2021, were used to predict the next 6 months.

Figure 4 illustrates the number of cumulative predicted infected population according to the reported statistics. Based on the model prediction the peak of the total number of infected population will occur around the end of May and start of June 2021, which the number of cases will gradually grow. To make the curve flatten, government could use interventions to reduce the number of cases and make it close to the threshold of hospital beds. By doing this, hospital over-admission could be avoided resulting in a decrease in the health staff workload. Despite the other countries, Iran has not declared any specific program to vaccinate the population, so it is vital to make proper predictions of the total number of infected people and make effective decisions to fight against the pandemic. 
Fig. 3 Daily new cases and deaths in Iran from February 19,2020 , to January 31 , 2021. The gray area represents the daily cases and the red parts are the daily deaths
Fig. 4 Prediction of the new wave of the COVID-19 in Iran. The blue line represents the daily cases and the red line corresponds to the best fit of the SEIR model to the actual data

\section{Daily COVID-19 cases and death of Iran}
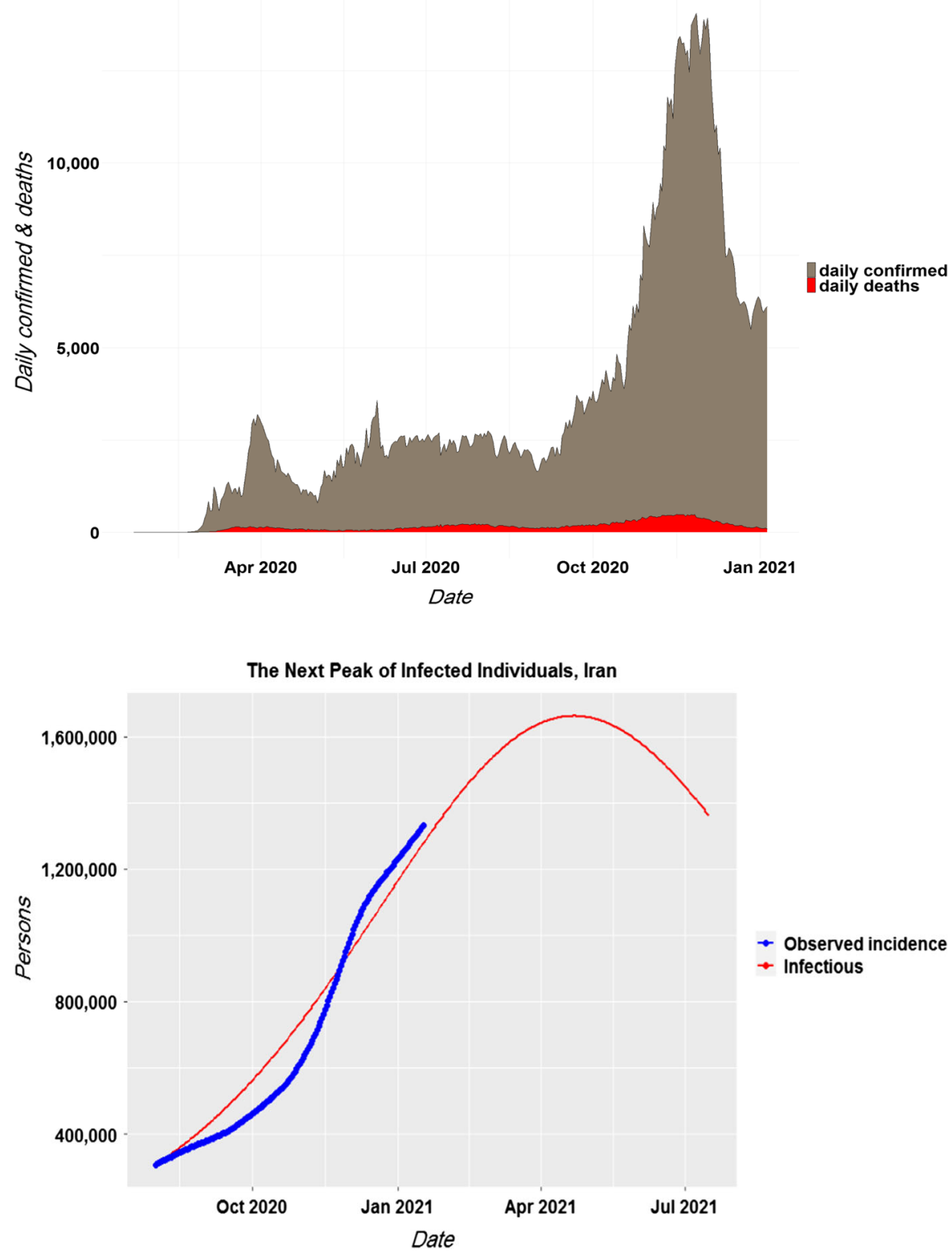

\section{Analysis and results}

The results and analysis of the applied interventions will be presented in the following sub-sections. The first sub-section is devoted to investigate the effectiveness of lockdown and its impacts. Some nonpharmaceutical interventions can be captured in the model using corresponding values of $\mathcal{R}_{0}$. SubSect. 4.2 explains the effect of different interventions and the results within the population.
4.1 Impact of implemented lockdowns on daily cases and deaths

In this section, the influence of implemented nonpharmaceutical interventions on epidemic spread will be analyzed. This analysis helps policymakers to better understand the impact of the implemented policy on the epidemic and the resulting number of new cases and deaths. Based on the impact of each implemented policy on $\mathcal{R}_{0}$ and the resulting new cases 
and death rate, the output of the analysis would be used in setting the new $\mathcal{R}_{0}$ values related to each new policy.

The first intervention to fight against pandemics in Iran was the Nowruz holiday lockdown. This intervention, which was applied on March 20th, 2020, included travel restrictions and closing welfare and recreational services for 14 days. Figure 5 illustrates the impact of this policy on the number of infected and mortality. It is assumed that the result of interventions appears about two weeks later so 14 days before and after each intervention were considered to observe and evaluate the result more efficiently. The lockdown applied from day 59 until the end of day 73. As it is shown in Fig. 5 the number of confirmed individuals reached its peak on day 69 which was 3186 and after that, the effect of lockdown appeared and it started to decrease. The amount of mortality follows the same pattern. After this wave, governmental offices, markets, and stores were opened. On July $5^{\text {th }}$, the COVID19 policy initiative committee made it mandatory to wear masks in offices, businesses, and crowded places.

The second lockdown was announced on October $3^{\text {rd }}$ for one week in seven provinces in red condition. Figure 6 demonstrates the changes before, after, and during this policy. According to the number of cases and deaths, this intervention has not made any significant difference and just kept the growth rate constant and after about 10 days the number of daily confirmed cases started to grow. About 17 days later on October 26th, the third intervention was applied to red cities from day 279 to day 286 for one week and the activities of the three business categories and occupational groups were limited. As it is shown in Fig. 7 this strategy could not reduce the infectious and death rate and the number of cases increased rapidly and passed 13,000 in one day and deaths reached 480.

Since the previous interventions were not effective and the number of hospitalizations was increasing speedily the authorities decided to use stricter interventions. From the start of November $21^{\text {st }}$, travel to and from red cities was banned and vehicles were not allowed to pass after 9 p.m. Figure 8 illustrates the impact of this strategy. After two weeks, the intervention showed its effectiveness and the number of cases and deaths started to decline steadily. Based on all four figures Iran has passed three waves so far of which the third one was the deadliest. According to the cases and death figures, Iran reported only the number of critical and hospitalized cases because the pick of deaths and cases is the same, but in theory, the pick of death should be one or two weeks after the pick of infected person.

\subsection{Impact of interventions on $\mathcal{R}_{0}$}

The basic reproduction number $\left(\mathcal{R}_{0}\right)$ denotes the number of individuals that can be infected by an
Fig. 5 Nowruz holiday (March 20 $0^{\text {th }}, 2020$, for 14 days) lockdown. Gray bars indicate the number of daily confirmed cases and the red areas demonstrate the number of daily deaths. Data source: (CSSE) at Johns Hopkins University

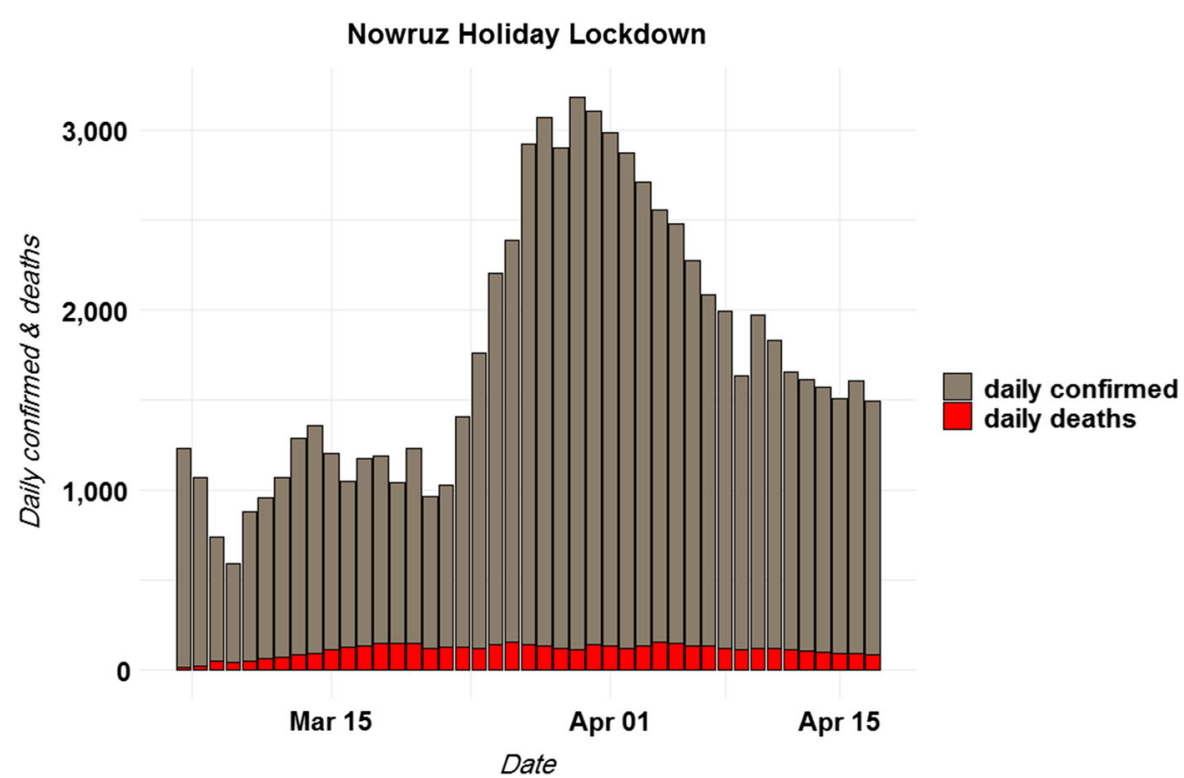


Fig. 6 October 3rd (a week length) lockdown. Gray bars indicate the number of daily confirmed cases and the red areas demonstrate the number of daily deaths. Data source: (CSSE) at Johns Hopkins University

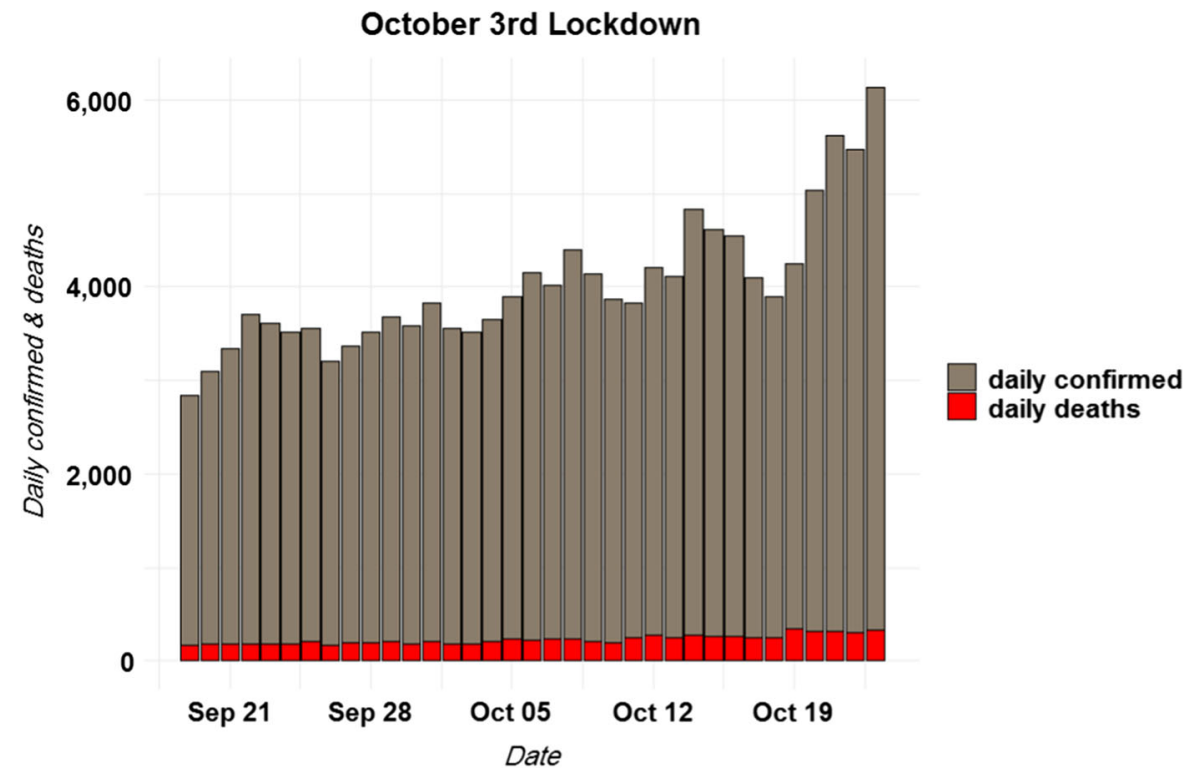

October 26th Lockdown

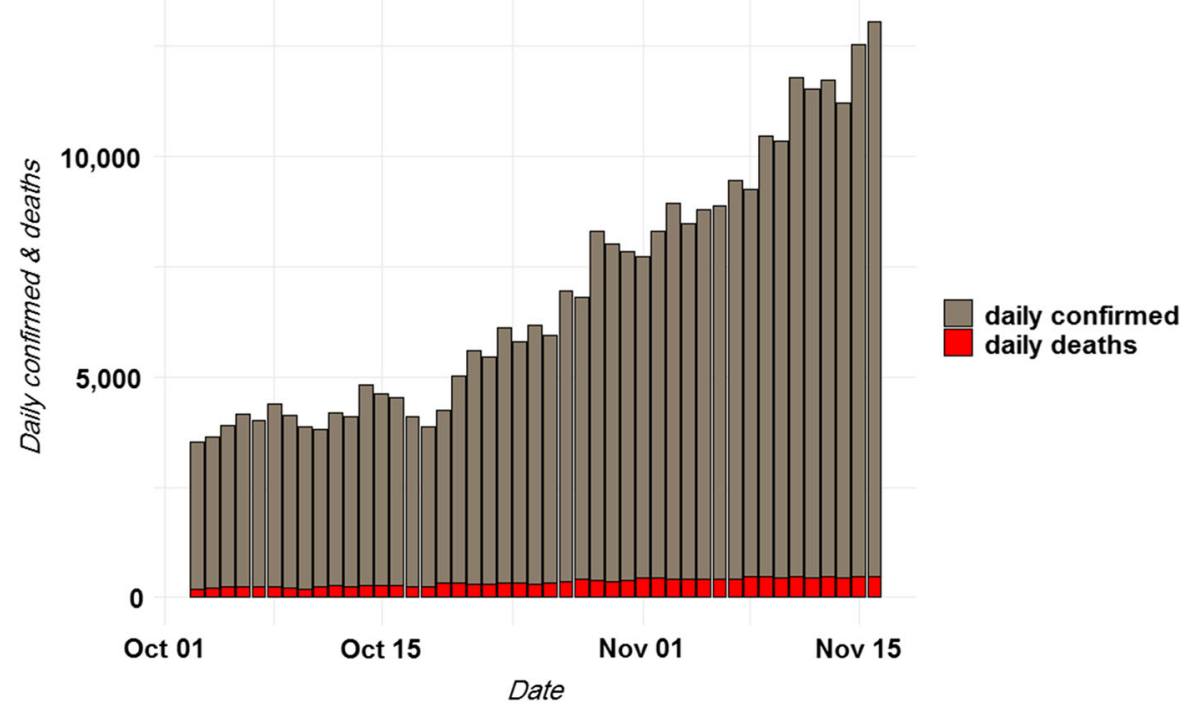

Fig. 7 October 26th (a week length) lockdown. Gray bars indicate the number of daily confirmed cases and the red areas demonstrate the number of daily deaths. Data source: (CSSE) at Johns Hopkins University

infected person. $\mathcal{R}_{0}$ is not affected only by viral characteristics but also by environmental conditions or in the case of COVID-19 by people contact rate. This makes it an important factor in evaluating policies and government' interventions in dealing with the current pandemic situation. Intervention strategies such as lockdown have a direct impact on people contact rate which can reduce the number of infected cases.
Figure 9 illustrates the estimated number of cases for Iran during the first 90 days of epidemic under various $\mathcal{R}_{0}$. As it is shown, the higher rates of $\mathcal{R}_{0}$, increase the number of cases exponentially. When the value of $\mathcal{R}_{0}$ becomes greater than three the number of infected people grows dramatically.

To estimate the impact of various intervention policies on the number of infected people in Iran, two 
Fig. 8 November 21st lockdown. Gray bars indicate the number of daily confirmed cases and the red areas demonstrate the number of daily deaths. Data source: (CSSE) at Johns Hopkins University
Fig. 9 Estimated number of cases for Iran with five different $\mathcal{R}_{0}$ for 90 days. Each line represents the number of cases corresponding to a specific value of $\mathcal{R}_{0}$. The $\mathrm{R} 0 \mathrm{~s}$ in the figure refers to the basic reproduction number $\left(\mathcal{R}_{0}\right)$

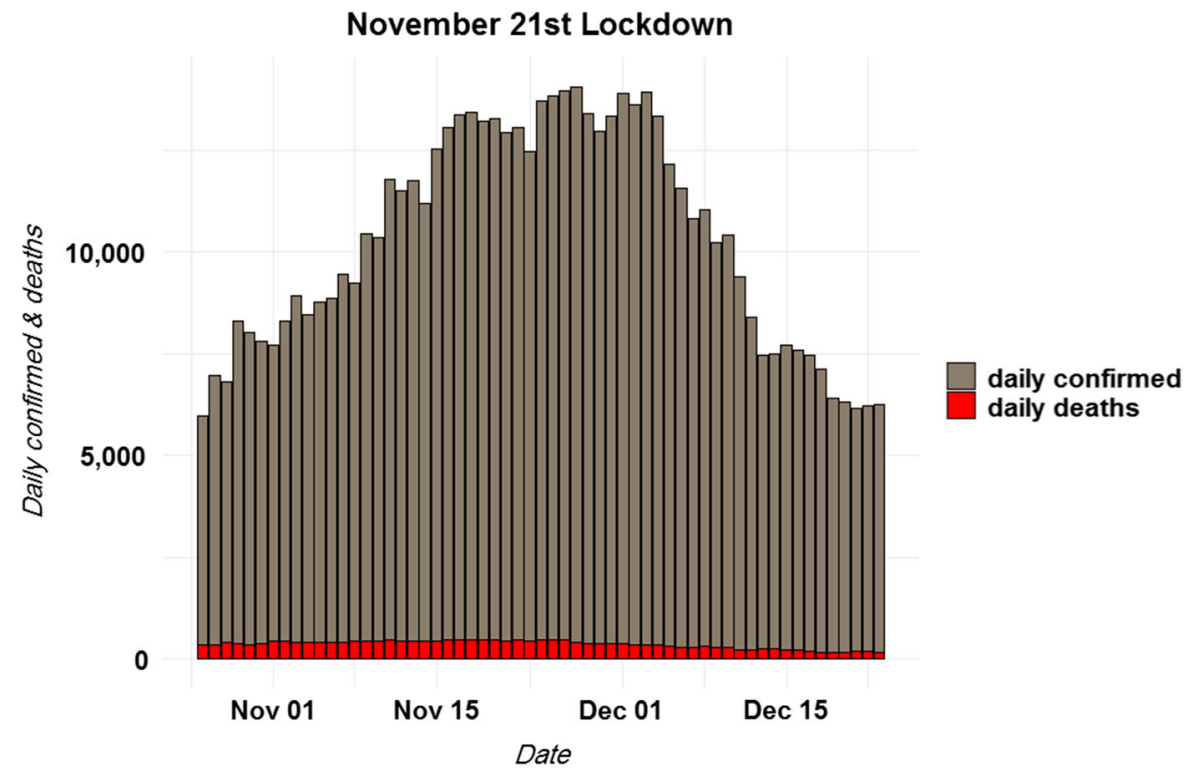

Estimated Number of Cases With Different R0 in the First 90 Days

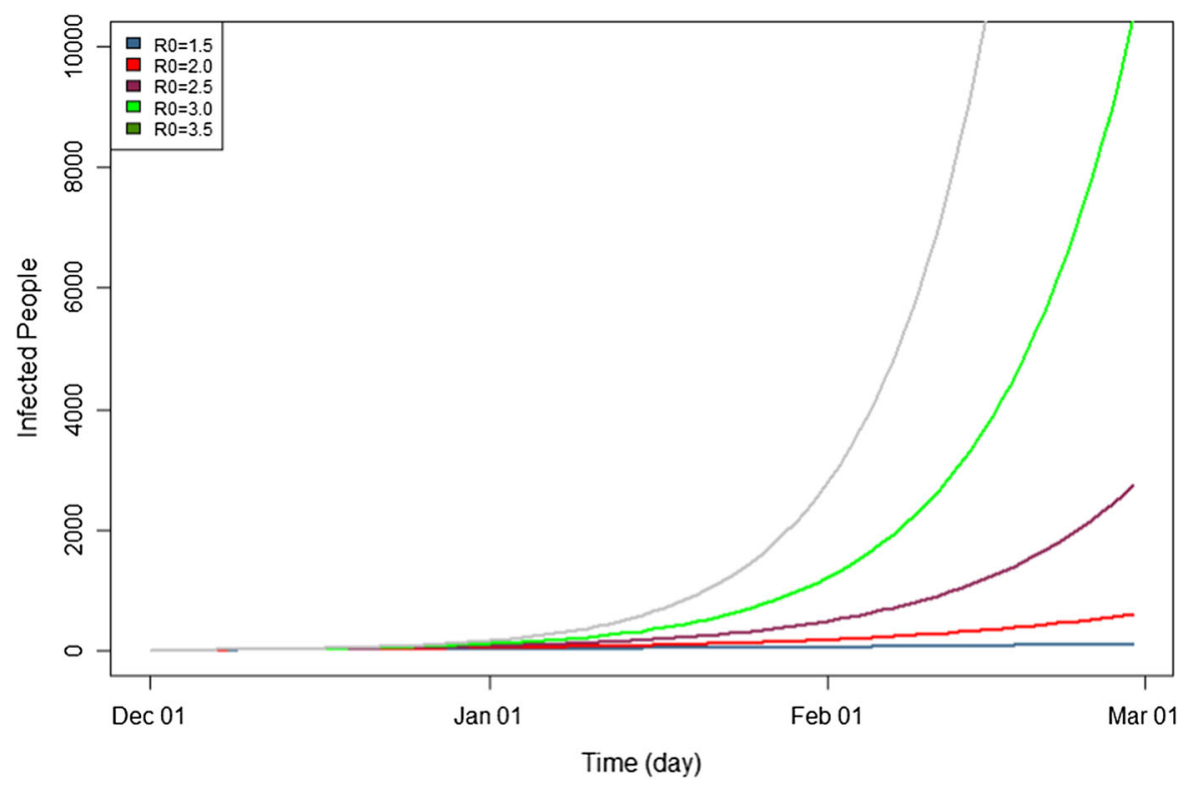

scenarios have been considered. In the first one, it is assumed that the number of exposed people is ten times the number of infected people. In the second one the number of exposed people is set to be 20 times the number of cases.

Figure 10 demonstrates the number of infected population in the first scenario with low number of exposed and Fig. 10 displays the number of sick people with high amount of exposure in the second scenario. The effect of government interventions on the number of cases from the beginning of the pandemic is shown with different $\mathcal{R}_{0}$ in Figs. 10 and 11. In the first scenario with high exposed rate, the number of infected people in the peak is more than $1,200,000$, while in the second scenario with the higher rate of exposed people, it reached just under $2,500,000$. The Nowruz holiday lockdown was the first hard intervention with $\mathcal{R}_{0}=1.2$. The line below the 
Fig. 10 Impact of interventions under the low value of $\mathrm{E}$ compartment. The R0s in the figure refers to the basic reproduction number $\left(\mathcal{R}_{0}\right)$
Fig. 11 Impact of interventions under the high value of E compartment. The R0s in the figure refers to the basic reproduction number $\left(\mathcal{R}_{0}\right)$
Impact with Low E $=10$ * I, with 2 Infected

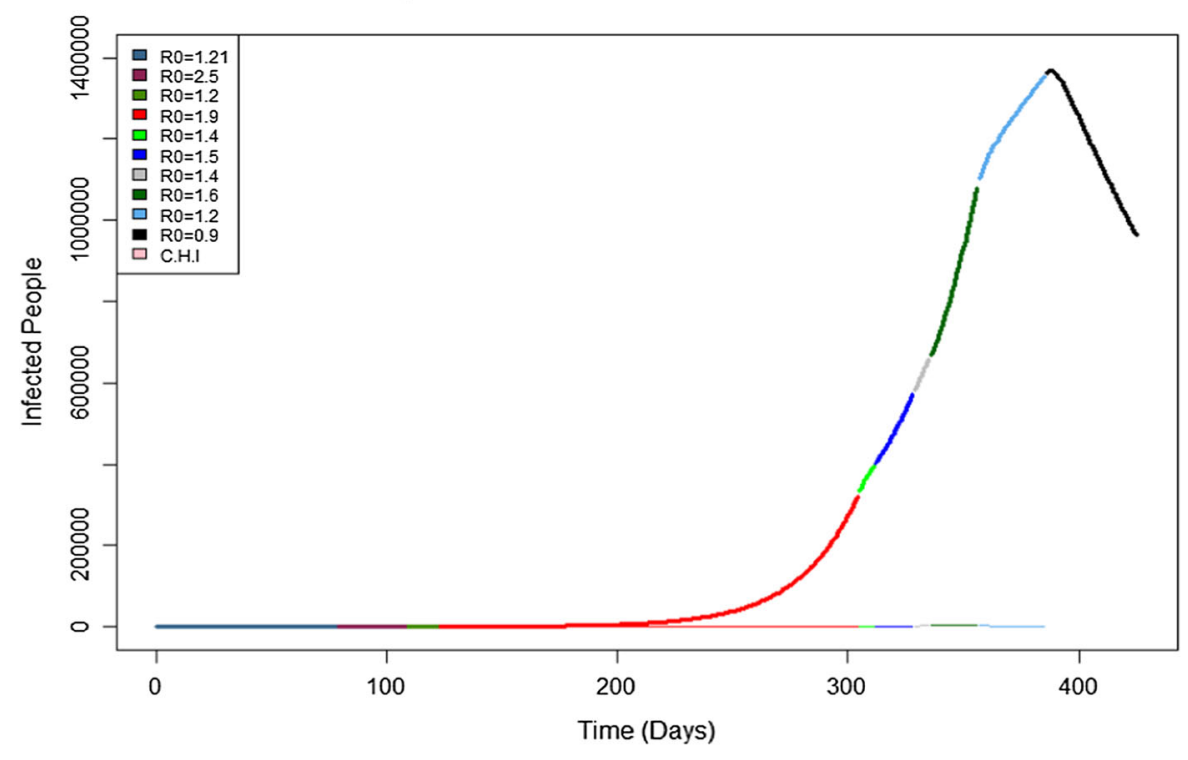

Impact with High E $=20$ *I

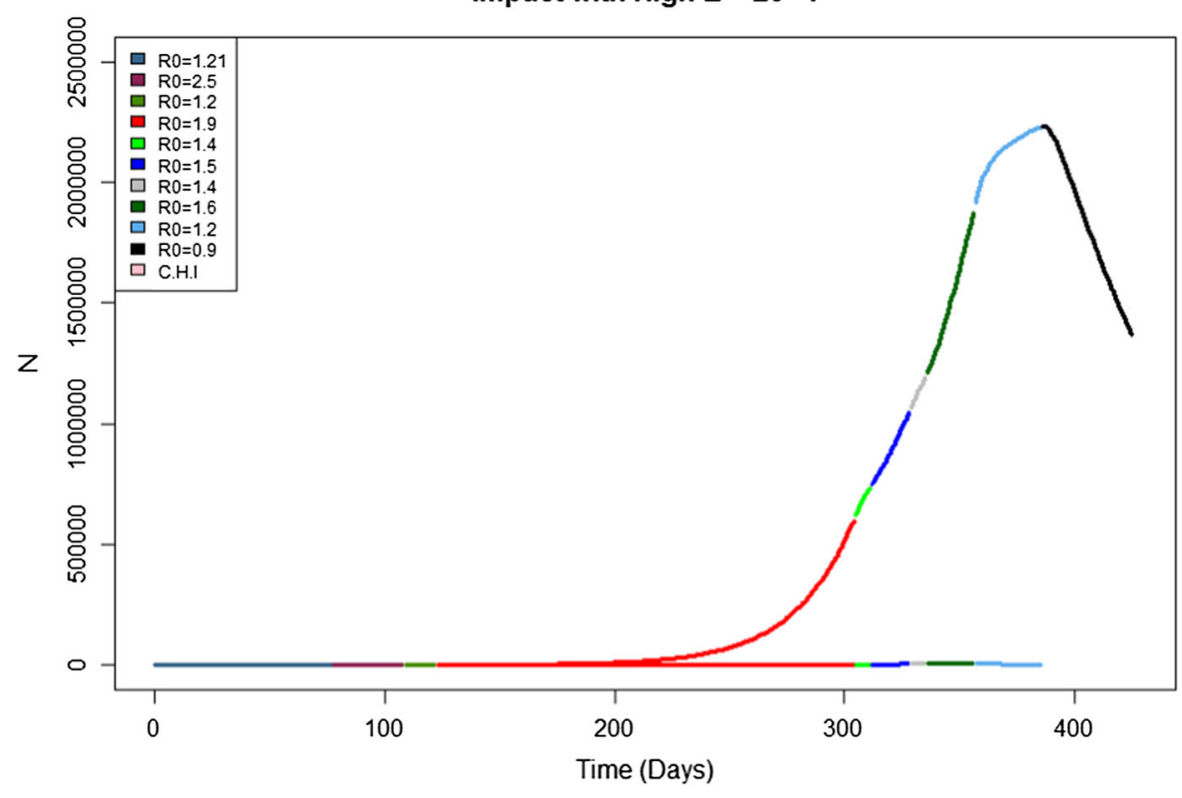

curves indicates the impact of this policy on the amount of infected people if government had decided to continue the policy. In reality, in spite of its great impact on the number of cases, this policy caused a lot of economic issues so policy makers decided to cancel it and limited their interventions to those cities which were in red condition. Because of an early release of all the restrictions, this intervention was not as effective as it was expected, resulting in a sharp increase in the number of people being infected.

\section{Conclusion}

In this paper, the impact of non-pharmaceutical interventions on the number of death and infected people in Iran was investigated. Since the beginning of 
the pandemic, the Iranian government applied various policies to control the epidemic. The Nowruz lockdown was one of the strictest interventions that led to economic issues, which forced the government to go for soft interventions with less restrictions. This intervention approach increased the amount of cases and caused a high peak in the number of infected people and death. In addition, the basic reproduction number, which is an important factor in evaluating the policies and controlling the spread of a virus, was analyzed with low and high number of exposed individuals. To predict the peak of the number of infected people, the mathematical SEIR model was used. The official reported data were applied to estimate the model parameters. The prediction revealed that the peak of infected population will occur around the end of May and start of June 2021; therefore, we suggest the officials to consider proper policies to reduce this number and avoid hospital overadmissions. This paper investigated the impact of nonpharmaceutical interventions. To capture the effects of vaccination, it is possible to include the transformation rate between the susceptible and recovered. The rate must be based on the true effect of the vaccine. Therefore, it is an interesting research agenda to investigate the effect of public vaccination on the epidemic.

Acknowledgements The authors would like to appreciate the anonymous reviewers' valuable and profound comments on an earlier version of the manuscript that have resulted in significant improvements to the article.

Funding This research has been funded by Persian Gulf University and the National Institute of Genetic Engineering and Biotechnology (NIGEB) of Iran under grant number 99-S-ZH392.

Data availability The datasets generated during and/or analyzed during the current study are publicly available in the Johns Hopkins, GitHub data repository.

\section{Declarations}

Conflict of interest The authors declare that they have no conflict of interest.

\section{References}

1. Erkoreka, A.: Epidemics in nortthern Basque: black death and the Spanish influenza. Hist. Sci. Med. 42(2), 113-22 (2008)

2. Ouassou, H., et al.: The pathogenesis of coronavirus disease 2019 (COVID-19): evaluation and prevention. J. Immunol. Res. 2020, 1-7 (2020). https://doi.org/10.1155/2020/ 1357983

3. WHO, WHO Director-General's opening remarks at the media briefing on COVID-19-11 March 2020. WHO, 2020. https://www.who.int/dg/speeches/detail/whodirector-general-s-opening-remarks-at-the-media-briefingon-covid-19-11-march-2020.

4. Smith, P.G., Morrow, R.H., Ross, D.A.: Types of intervention and their development, 3 edn. In: Field Trials of Health Interventions: A Toolbox. Oxford University Press, Oxford (2015)

5. Yáñez, F.G., Andrea, C.H.:Towards the control of epidemic spread: designing reinforcement learning environments. In: AICS, pp. 188-199, 2019

6. Ebrahim, S.H., Ahmed, Q.A., Gozzer, E., Schlagenhauf, P., Memish, Z.A.: Covid-19 and community mitigation strategies in a pandemic. BMJ (2020). https://doi.org/10.1136/ bmj.m1066

7. Merriam-Webster, lockdown. 2021. https://www.merriamwebster.com/dictionary/lockdown (Accessed Jan. 20, 2021).

8. Jehan Al Humaid, F.A.H., Khan, S.Q., Farooqi, A.A., Alhareky, M., Alonaizan, F.: COVID-19: Impact of early decision and type of lockdown over the spread of the virus," 2020. [Online]. Available: https://www.researchsquare. com/article/rs-26573/v1.

9. Sardar, T., Nadim, S.S., Rana, S., Chattopadhyay, J.: Assessment of lockdown effect in some states and overall India: a predictive mathematical study on COVID-19 outbreak. Chaos, Solitons Fractals 139, 110078 (2020). https:// doi.org/10.1016/j.chaos.2020.110078

10. Giordano, G., et al.: Modelling the COVID-19 epidemic and implementation of population-wide interventions in Italy. Nat. Med. 26(6), 855-860 (2020). https://doi.org/10.1038/ s41591-020-0883-7

11. Liu, M., Thomadsen, R., Yao, S.: Forecasting the spread of COVID-19 under different reopening strategies. Sci. Rep. 10(1), 20367 (2020). https://doi.org/10.1038/s41598-02077292-8

12. Kudryashov, N.A., Chmykhov, M.A., Vigdorowitsch, M.: Analytical features of the SIR model and their applications to COVID-19. Appl. Math. Model. 90, 466-473 (2021). https://doi.org/10.1016/j.apm.2020.08.057

13. Malavika, B., Marimuthu, S., Joy, M., Nadaraj, A., Asirvatham, E.S., Jeyaseelan, L.: Forecasting COVID-19 epi- 
demic in India and high incidence states using SIR and logistic growth models. Clin. Epidemiol. Glob. Heal. 9, 26-33 (2021). https://doi.org/10.1016/j.cegh.2020.06.006

14. Alanazi, S.A., Kamruzzaman, M.M., Alruwaili, M., Alshammari, N., Alqahtani, S.A., Karime, A.: Measuring and preventing COVID-19 using the SIR model and machine learning in smart health care. J. Healthc. Eng. 2020, 1-12 (2020). https://doi.org/10.1155/2020/8857346

15. Sadurní, E., Luna-Acosta, G.: Exactly solvable SIR models, their extensions and their application to sensitive pandemic forecasting. Nonlinear Dyn. 103(3), 2955-2971 (2021). https://doi.org/10.1007/s11071-021-06248-y

16. Ghanbari, B.: On forecasting the spread of the COVID-19 in Iran: the second wave. Chaos, Solitons Fract. 140, 110176 (2020). https://doi.org/10.1016/j.chaos.2020.110176

17. Tutsoy, O., Colak, S., Polat, A., Balikci, K.: A novel parametric model for the prediction and analysis of the COVID19 casualties. IEEE Access 8, 193898-193906 (2020). https://doi.org/10.1109/ACCESS.2020.3033146

18. Huang, J., et al.: Global prediction system for COVID-19 pandemic. Sci. Bull. 65(22), 1884-1887 (2020). https://doi. org/10.1016/j.scib.2020.08.002

19. Wangping, J., et al.: Extended SIR prediction of the epidemics trend of COVID-19 in Italy and compared with Hunan, China. Front. Med. (2020). https://doi.org/10.3389/ fmed.2020.00169

20. Das, S.: Prediction of COVID-19 disease progression in India: under the effect of national lockdown. Apr. 2020, [Online]. Available: http://arxiv.org/abs/2004.03147.

21. Qi, C., Karlsson, D., Sallmen, K., Wyss, R.: Model studies on the COVID-19 pandemic in Sweden. Apr. 2020, [Online]. Available: http://arxiv.org/abs/2004.01575.

22. Rădulescu, A., Williams, C., Cavanagh, K.: Management strategies in a SEIR-type model of COVID 19 community spread. Sci. Rep. 10(1), 21256 (2020). https://doi.org/10. 1038/s41598-020-77628-4

23. Kwuimy, C.A.K., Nazari, F., Jiao, X., Rohani, P., Nataraj, C.: Nonlinear dynamic analysis of an epidemiological model for COVID-19 including public behavior and government action. Nonlinear Dyn. 101(3), 1545-1559 (2020). https://doi.org/10.1007/s11071-020-05815-z

24. He, S., Peng, Y., Sun, K.: SEIR modeling of the COVID-19 and its dynamics. Nonlinear Dyn. 101(3), 1667-1680 (2020). https://doi.org/10.1007/s11071-020-05743-y

25. Xu, C., Yu, Y., Chen, Y., Lu, Z.: Forecast analysis of the epidemics trend of COVID-19 in the USA by a generalized fractional-order SEIR model. Nonlinear Dyn. 101(3), 1621-1634 (2020). https://doi.org/10.1007/s11071-02005946-3

26. Arcede, J.P., Caga-anan, R.L., Mentuda, C.Q., Mammeri, Y.: Accounting for symptomatic and asymptomatic in a SEIR-type model of COVID-19. Math. Model. Nat. Phenom. 15, 34 (2020). https://doi.org/10.1051/mmnp/2020021

27. Radha, M., Balamuralitharan, S.: A study on COVID-19 transmission dynamics: stability analysis of SEIR model with Hopf bifurcation for effect of time delay. Adv. Differ. Eq. 2020(1), 523 (2020). https://doi.org/10.1186/s13662020-02958-6

28. Das, A., Dhar, A., Goyal, S., Kundu, A., Pandey, S.: COVID-19 Analytic results for a modified SEIR model and comparison of different intervention strategies. Chaos
Solitons Fract. 144, 110595 (2020). https://doi.org/10.1016/ j.chaos.2020.110595

29. Wikipedia, COVID-19 pandemic in Iran. 2020. https://en. wikipedia.org/wiki/COVID-19_pandemic_in_Iran.

30. Brauer, F.: Mathematical epidemiology: past, present, and future. Infect. Dis. Model. 2(2), 113-127 (2017). https://doi. org/10.1016/j.idm.2017.02.001

31. Ameri, K., Cooper, K.D.: A network-based compartmental model for the spread of whooping cough in Nebraska. AMIA Summits Transl Sci Proc 2019, 388 (2019)

32. Kermack, W.O., McKendrick, A.G.: Contributions to the mathematical theory of epidemics-I. Bull. Math. Biol. 53(1-2), 33-55 (1991). https://doi.org/10.1007/ BF02464423

33. Padmanabhan, P., Seshaiyer, P.: Computational and mathematical methods to estimate the basic reproduction number and final size for single-stage and multistage progression disease models for zika with preventative measures. Comput. Math. Methods Med. 2017, 1-17 (2017). https://doi. org/10.1155/2017/4290825

34. Dong, E., Du, H., Gardner, L.: An interactive web-based dashboard to track COVID-19 in real time. Lancet Infect. Dis. 20(5), 533-534 (2020). https://doi.org/10.1016/S14733099(20)30120-1

35. Liu, X.-X., Fong, S.J., Dey, N., Crespo, R.G., HerreraViedma, E.: A new SEAIRD pandemic prediction model with clinical and epidemiological data analysis on COVID19 outbreak. Appl. Intell. (2021). https://doi.org/10.1007/ s10489-020-01938-3

36. Petzold, L.: Automatic selection of methods for solving stiff and nonstiff systems of ordinary differential equations. SIAM J. Sci. Stat. Comput. 4(1), 136-148 (1983). https:// doi.org/10.1137/0904010

37. Hindmarsh, A.C., Petzold, L.R.: Algorithms and software for ordinary differential equations and differential- algebraic equations, Part II: Higher-order methods and software packages. Comput. Phys. 9(2), 148 (1995). https://doi.org/ 10.1063/1.168540

38. Nocedal, J.: Updating quasi-Newton matrices with limited storage. Math. Comput. 35(151), 773-773 (1980). https:// doi.org/10.1090/S0025-5718-1980-0572855-7

39. Saputro, D.R.S., Widyaningsih, P.: Limited memory Broyden-Fletcher-Goldfarb-Shanno (L-BFGS) method for the parameter estimation on geographically weighted ordinal logistic regression model (GWOLR). AIP Conf. Proc. 1868, 040009 (2017). https://doi.org/10.1063/1.4995124

40. Morales, J.L., Nocedal, J.: Remark on 'algorithm 778: L-BFGS-B: Fortran subroutines for large-scale bound constrained optimization. ACM Trans. Math. Softw. 38(1), 1-4 (2011). https://doi.org/10.1145/2049662.2049669

41. Zhu, C., Byrd, R.H., Lu, P., Nocedal, J.: Algorithm 778: L-BFGS-B. ACM Trans. Math. Softw. 23(4), 550-560 (1997). https://doi.org/10.1145/279232.279236

42. Byrd, R.H., Lu, P., Nocedal, J., Zhu, C.: A limited memory algorithm for bound constrained optimization. SIAM J. Sci. Comput. 16(5), 1190-1208 (1995). https://doi.org/10.1137/ 0916069

43. Alenezi, M.N., Al-Anzi, F.S., Alabdulrazzaq, H., Alhusaini, A., Al-Anzi, A.F.: A study on the efficiency of the estimation models of COVID-19. Results Phys. 26, 104370 (2021). https://doi.org/10.1016/j.rinp.2021.104370 
44. Abou-Ismail, A.: Compartmental models of the COVID-19 pandemic for physicians and physician-scientists. SN Compr. Clin. Med. 2(7), 852-858 (2020). https://doi.org/10. 1007/s42399-020-00330-z

45. WHO, Transmission of SARS-CoV-2: implications for infection prevention precautions. World Health Organization, 2020. https://www.who.int/news-room/commentaries/ detail/transmission-of-sars-cov-2-implications-forinfection-prevention-precautions (accessed Mar. 29, 2020).

Publisher's Note Springer Nature remains neutral with regard to jurisdictional claims in published maps and institutional affiliations. 